\title{
Treatment of Metastatic Extramammary Paget Disease with Combination Ipilimumab and Nivolumab: A Case Report
}

\author{
Brendan John Guercio ${ }^{a}$ Gopa lyer ${ }^{a}$ b Wajih Zaheer Kidwai ${ }^{c}$ \\ Mario E. Lacouture ${ }^{a}$ d Soleen Ghafoore Anthony M. Rossia, ${ }^{\mathrm{a}}$ \\ David N. Assis ${ }^{f} \quad$ Ying-Bei Chen ${ }^{g, h} \quad$ Klaus J. Busamg, h Yelena \\ Y. Janjigian ${ }^{a, b}$ Komal Jhaveria, b Darren R. Feldman ${ }^{a, b} \quad$ Anne Capozzi $^{a}$ \\ Vanessa Figueroa $^{a}$ Dean F. Bajorin ${ }^{a, b}$ Jonathan E. Rosenberg ${ }^{a, b}$ \\ Travis J. Hollmann ${ }^{g}$, h Samuel A. Funt ${ }^{a}$ b \\ aDepartment of Medicine, Memorial Sloan Kettering Cancer Center, New York, NY, USA; \\ ${ }^{b}$ Department of Medicine, Weill Cornell Medicine, New York, NY, USA; 'Department of \\ Medicine, Yale Cancer Center/Smilow Cancer Hospital, New Haven, CT, USA; ${ }^{\mathrm{d} D e p a r t m e n t}$ \\ of Dermatology, Weill Cornell Medicine, New York, NY, USA; eInstitute of Diagnostic and \\ Interventional Radiology, University Hospital Zurich, Zurich, Switzerland; ${ }^{f}$ Department \\ of Medicine, Yale School of Medicine, New Haven, CT, USA; 9Department of Pathology,

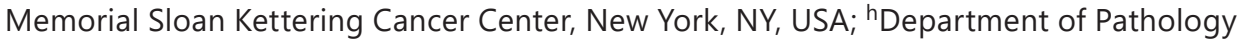 \\ and Laboratory Medicine, Weill Cornell Medicine, New York, NY, USA
}

\section{Keywords}

Extramammary Paget disease $\cdot$ Immunotherapy $\cdot$ Nivolumab $\cdot$ Ipilimumab $\cdot$ Immune checkpoint blockade

\begin{abstract}
Metastatic primary cutaneous extramammary Paget disease (EMPD) is a rare clinical entity with a 5-year survival $<10 \%$ and no standard therapy. We report the first case to our knowledge of metastatic EMPD with treatment response to checkpoint inhibitor immunotherapy. The patient had diffusely metastatic disease and previously progressed on cytotoxic chemotherapy and a molecularly targeted agent. Treatment with four cycles of ipilimumab $1 \mathrm{mg} / \mathrm{kg}$ plus nivolumab $3 \mathrm{mg} / \mathrm{kg}$ resulted in a durable partial response lasting 7 months. Analysis of metastatic tumor tissue failed to identify known predictors of treatment response to immune checkpoint inhibitors, such as high PD-L1 expression, high tumor mutation burden, or microsatellite instability. These findings support further investigation of immune checkpoint inhibition for the management of metastatic EMPD, which currently has an abysmal prognosis and no standard therapies.




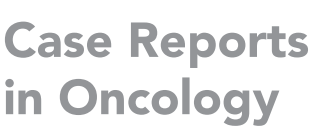

\section{Introduction}

Primary cutaneous extramammary Paget disease (EMPD) is a rare cutaneous adenocarcinoma usually originating in the anogenital area or axillae [1]. Primary cutaneous EMPD is thought to arise from apocrine or eccrine glands or possibly from pluripotent stem cells or Toker cells [2]. EMPD tends to be indolent and is often identified as carcinoma in situ at the time of diagnosis [1]. However, when distant metastases occur, 5-year survival rates are $<10 \%$ and there is no standard therapy [1,3]. We report the first case to our knowledge of metastatic EMPD with durable response to checkpoint inhibitor immunotherapy. Following the patient's passing, written informed consent was obtained from the patient's next of kin for the publication of this case report and any accompanying images.

\section{Case Presentation}

A 55-year-old man was found to have an elevated PSA of $6.5 \mathrm{ng} / \mathrm{mL}$ and underwent magnetic resonance imaging of the pelvis, revealing a $2.5 \mathrm{-cm}$ lesion in the prostate as well as extensive neoplasm in the bladder, accompanied by osseous metastases, adenopathy, and left ureteral obstruction. Computed tomography (CT) of the chest, abdomen and pelvis revealed diffuse bony metastases of the spine, ribs, and pelvis, as well as lymphadenopathy of the thorax, pelvis and retroperitoneum. The patient underwent concurrent bladder and prostate biopsies, which revealed extensive involvement of the bladder wall and prostate by an infiltrating, poorly differentiated carcinoma consisting of solid nests or cords of tumor cells with prominent nucleoli and abundant eosinophilic and granular cytoplasm. There was no urothelial carcinoma in situ or typical prostate adenocarcinoma identified. Immunohistochemical studies showed that the tumor cells were positive for GATA3, CK903, androgen receptor (diffuse, strong) and HER2 (2+), and negative for p63, PSA, PSAP, PSMA, NKX3.1, synaptophysin, chromogranin, ER and PR. A longstanding rash of the scrotum, which predated the patient's cancer diagnosis by 7 years, was subsequently biopsied. This revealed adenocarcinoma in the epidermis and dermis. The dermal component showed similar morphology to the infiltrating tumor involving both bladder and prostate. Immunohistochemically, these tumor cells were positive for CK903, GATA3, and CK7, and showed weak staining for Her2Neu $(1+)$. The clinical picture was thought to be consistent with widely metastatic EMPD of the scrotum.

Given the patient's excellent performance status, he underwent treatment with 13 cycles of modified DCF (docetaxel, cisplatin, 5-fluorouracil). This regimen, previously studied in the context of metastatic gastric cancer [4], was selected given prior reports of treatment response in EMPD to the combination's individual agents $[1,3]$. The patient achieved a partial response and his regimen was ultimately dose-reduced to 5-FU monotherapy for an additional 7 cycles. However, subsequent imaging revealed progression of disease including multiple liver metastases.

Next generation sequencing of the metastatic tumor specimen with a mean coverage of 625X using the FDA-authorized platform MSK-IMPACT [5] revealed microsatellite stability and alteration of PIK3CA exon 10 p.E545K (c.1633 G > A) with a mean allele frequency of $37.8 \%$, among other genetic alterations (shown in Table 1). The tumor mutation burden (TMB) was 4.4 mutations per megabase (mut/MB), compared to an average of 3.9 mut/MB for all cancer patient samples assessed by MSK-IMPACT. Given the somatic alteration in PIK3CA, treatment with alpelisib, an oral $\alpha$-specific PI3 kinase inhibitor, was initiated [6]. This was quickly complicated by known toxicities of alpelisib including nausea, grade 3 rash, and hyperglycemia as well as progression of disease, resulting in treatment discontinuation.

\section{Karger'}


Table 1. Somatic genetic alterations detected in the metastatic extramammary Paget disease tumor specimen

\begin{tabular}{|c|c|c|c|c|c|c|}
\hline Gene & Type & Alteration & Location & $\begin{array}{l}\text { NCBI Ref. } \\
\text { Sequence }\end{array}$ & $\begin{array}{l}\text { Mutant allele } \\
\text { frequency }\end{array}$ & $\begin{array}{l}\text { Fold } \\
\text { change }\end{array}$ \\
\hline PIK3CA & Missense mutation & p.E545K ${ }^{1}($ c.1633 G > A) & Exon 10 & NM_006218 & $37.8 \%$ & $\mathrm{~N} / \mathrm{A}$ \\
\hline KMT2C (MLL3) & Splicing mutation & p.X4632_splice $($ c. $13894+2 \mathrm{~T}>\mathrm{C}$ ) & Exon 52 & NM_170606 & $29.3 \%$ & $\mathrm{~N} / \mathrm{A}$ \\
\hline HIST1H3D & Missense mutation & p.Y55 N (c.163T > A) & Exon 2 & NM_003530 & $27.5 \%$ & $\mathrm{~N} / \mathrm{A}$ \\
\hline POLE & Missense mutation & p.N1994I (c.5981A > T) & Exon 43 & NM_006231 & $26.5 \%$ & $\mathrm{~N} / \mathrm{A}$ \\
\hline STAT5A & Missense mutation & p.E73K (c.217 G > A) & Exon 4 & NM_003152 & $24.1 \%$ & $\mathrm{~N} / \mathrm{A}$ \\
\hline$C D K N 2 A / B$ & Whole gene deletion & Deletion & $9 p 21.3$ & $\begin{array}{l}\text { NM_004936 } \\
\text { NM_000077 } \\
\text { NM_058195 }\end{array}$ & $\mathrm{N} / \mathrm{A}$ & -2.0 \\
\hline PLK2 & Intragenic deletion & c.*3334_c.1156+48del & Exons 9-14 & NM_006622 & $\mathrm{N} / \mathrm{A}$ & $\mathrm{N} / \mathrm{A}$ \\
\hline
\end{tabular}

\footnotetext{
${ }^{1}$ Hotspot mutation.
}

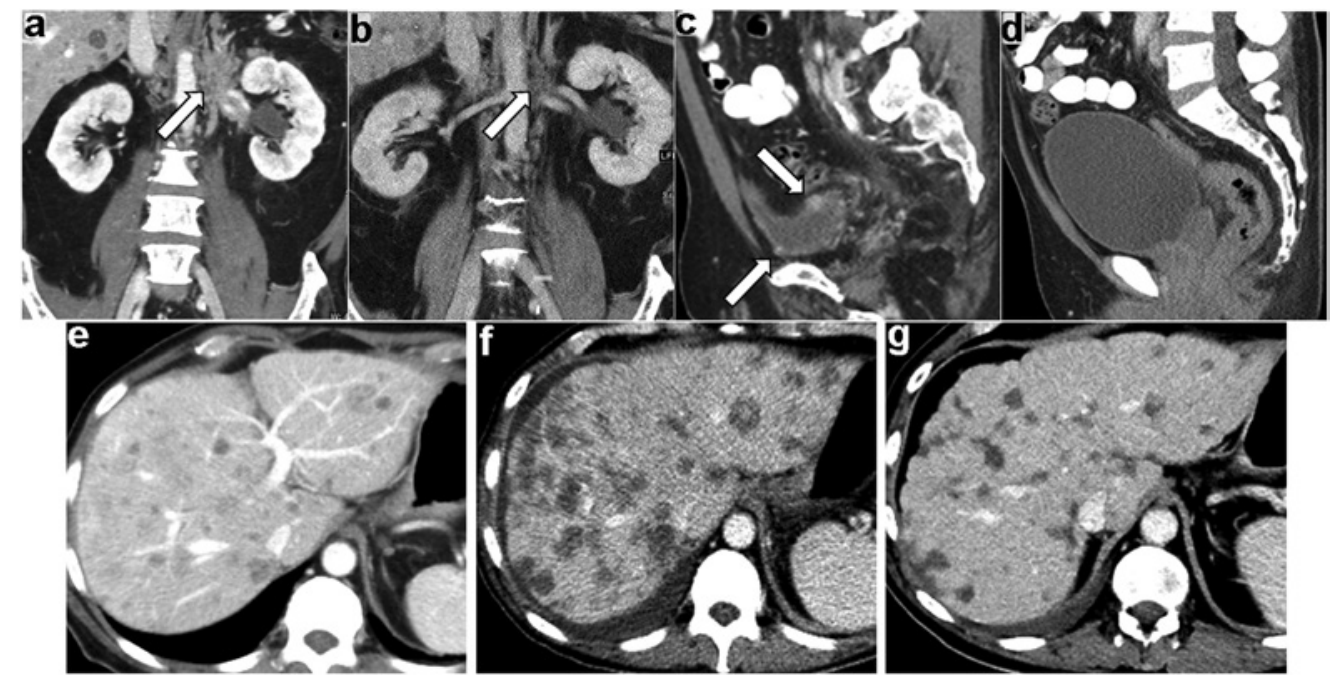

Fig. 1. Coronal contrast-enhanced CT showing infiltrative retroperitoneal metastasis (a) prior to initiation of ipilimumab and nivolumab compared to 5 months after immunotherapy initiation (b). Sagittal contrastenhanced CT shows hypervascular tumor implants on the bladder (c) prior to initiation of ipilimumab and nivolumab, which resolved 5 months after immunotherapy initiation (d). Axial contrast-enhanced CT depicts diffuse liver metastases (e) prior to initiation of ipilimumab and nivolumab compared to pseudoprogression 2 months after initiation of ipilimumab (f) plus nivolumab followed by partial response (g) as shown 5 months after immunotherapy initiation with decrease in number and enhancement of the lesions.

Treatment with ipilimumab $1 \mathrm{mg} / \mathrm{kg}$ plus nivolumab $3 \mathrm{mg} / \mathrm{kg}$ was then initiated, administered every 3 weeks. CT imaging after three cycles revealed partial treatment response, with decreased size of metastases in the bladder, decreased thoracoabdominal adenopathy, and findings in the liver that were later determined to be pseudoprogression (shown in Fig. 1). After cycle four, the patient developed immunotherapy-related hepatitis requiring systemic corticosteroids and mycophenolate mofetil, prohibiting further immunotherapy. Restaging CT scans 5 months after treatment initiation revealed further decrease in hepatic metastases, thoracoabdominal adenopathy, peritoneal carcinomatosis, and urinary bladder implants (shown in Fig. 1), as well as grossly stable osseous metastases. Scans 7 months after 


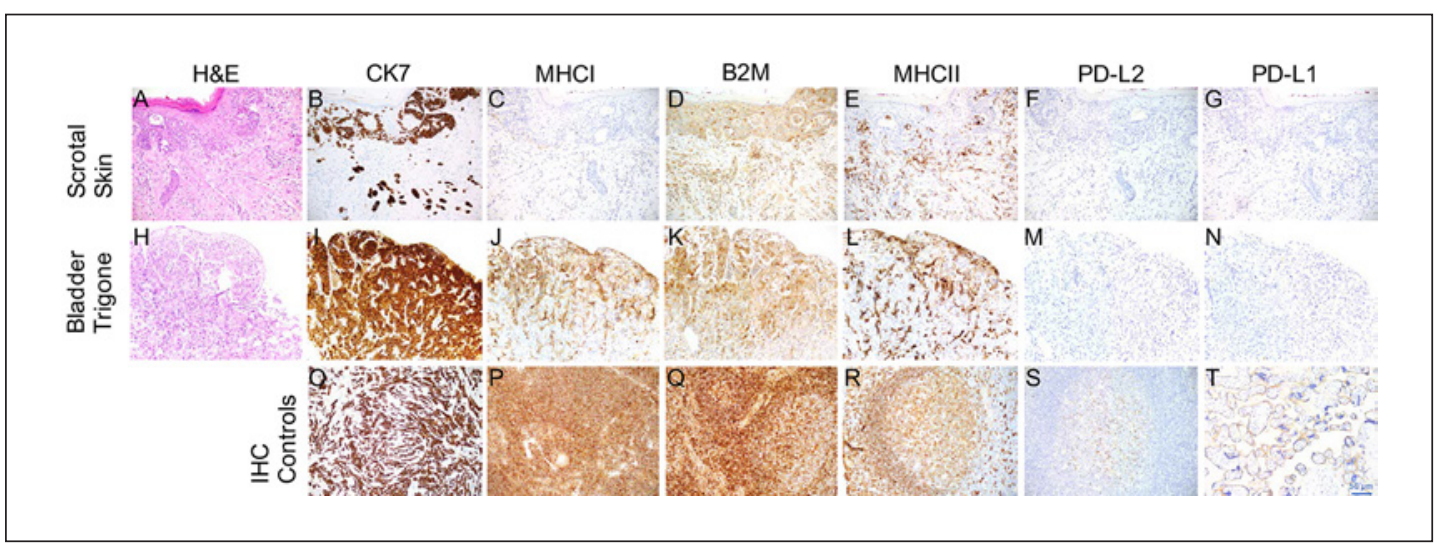

Fig. 2. Extramammary Paget disease in the scrotal skin $(\mathbf{A}-\mathbf{G})$ and bladder trigone $(\mathbf{H}-\mathbf{N})$ stains with CK7 (B, I) with minimal tumor expression of major histocompatibility complex class I (MHCI) (C, J), predominantly cytoplasmic tumor beta-2 microglobulin (B2M) (D, K), no tumor expression of major histocompatibility complex class II (MHCII) (E, L) and no tumor expression of programmed death-ligand 2 (PD-L2) (F, M) or programmed death-ligand 1 (PD-L1) (G, N) with minimal expression in the tumor microenvironment. Control immunohistochemistry (IHC) is shown using breast carcinoma for CK7 (O), lymph node for MHCI (P), B2M (Q), MHCII (R) and PD-L2 (S) and placenta for PD-L1 (T). All images were taken at $\times 20$. A $50 \mu \mathrm{m}$ scale bar is shown in $\mathbf{T}$.

treatment initiation ultimately demonstrated disease progression. The patient was rechallenged with nivolumab but did not respond and passed away due to disease progression. Immunohistochemical staining of the patient's pre-treatment metastatic bladder implant revealed absence of programmed death-ligand 1 (PD-L1) and PD-L2 expression by tumor cells, with minimal PD-L1 in the tumor microenvironment (shown in Fig. 2). Immunostaining of tumor cells revealed predominantly cytoplasmic expression of beta-2 microglobulin and minimal surface expression of major histocompatibility complex (MHC) class I.

\section{Discussion}

While localized EMPD is often successfully managed surgically, the prognosis for metastatic EMPD is poor [1,3]. Given the rarity of primary cutaneous metastatic EMPD, data for systemic therapy is generally limited to case reports and series with no standard treatment regimen [1,3]. Cytotoxic regimens reported in the literature include various chemotherapeutic agents alone or in combination, including cisplatin, docetaxel, 5-fluorouracil, paclitaxel, S-1, carboplatin, mitomycin-C, epirubicin, vincristine, and irinotecan [1, 3]. As in the case above, initial responses to chemotherapy can be positive but are ultimately transient and non-curative. Response rates generally range from 50 to $60 \%$, with median progression-free survival of 5-7 months and median overall survival $<17$ months [3]. Based on similarities to breast cancer, HER2-targeted therapies have been investigated in EMPD and case reports have demonstrated efficacy of trastuzumab [3]. Androgen receptor expression has also been identified in EMPD, and a single case report demonstrated treatment response in metastatic EMPD to bicalutamide plus leuprolide acetate [3]. Previous reports of EMPD have identified mutations of PIK3CA, AKT1, and the RAS/RAF pathway [3], but to our knowledge there is no literature to date establishing these as effective therapeutic targets in EMPD. Notably, our patient's disease did not respond to the PI3 kinase inhibitor alpelisib despite identification of a PIK3CA driver mutation, although drug delivery was affected by significant toxicity. 
Investigation of systemic immunotherapy for metastatic EMPD is supported by prior reports of effective management of localized EMPD with imiquimod, a topical Toll-like receptor 7 agonist which mediates anti-tumor response via immune cell activation with proinflammatory cytokines [2]. Systemic immunotherapies also feature prominently in the management of other cutaneous malignancies, and have even shown efficacy in rare cutaneous cancers such as Merkel cell carcinoma and sebaceous carcinoma [3, 7]. Consistent with our case findings, prior studies have shown low rates of PD-L1 expression by EMPD tumor cells, though PD-L1 expression in the tumor microenvironment has been described [8-11]. In the largest study to date examining samples from 48 patients, only $10 \%$ of invasive EMPD cases expressed PD-L1, though focal PD-L1 expression in tumor-infiltrating lymphocytes was identified in $72 \%$ of all EMPD cases [8]. Eighty-nine percent and 79\% expressed beta-2-microglobulin and MHC class I, respectively, which are required for antigen presentation and associated with immune checkpoint inhibitor response $[8,12]$. A separate study of 22 EMPD cases identified variable expression of CTLA-4 in 23\% of cases; CD3-positive T cells were present in nearly all specimens, though there was no relationship between CTLA-4 and CD3 positivity [10].

Though PD-L1 expression correlates with response to PD-1/PD-L1-targeted therapies in some settings, it often fails to predict treatment response [7]. Other predictors of response across various tumor types include microsatellite instability (MSI) and high TMB $[7,13]$. Our patient's tumor responded to immune checkpoint blockade despite microsatellite stability. While the tumor in this case demonstrated no evidence of MSI, a prior study identified germline mismatch repair gene missense mutations in 8 of 20 EMPD tumors (40\%), with 4 cases exhibiting MSI, lending rationale for consideration of immunotherapy in such cases [14]. Our patient's tumor demonstrated a roughly average TMB of $4.4 \mathrm{mut} / \mathrm{MB}$ when compared to all other tumors sequenced by MSK-IMPACT, which average 3.9 mut/MB [15]. This comparison suggests that our patient's response to immune checkpoint inhibitors was not clearly attributable to TMB, though levels of TMB predictive of immune checkpoint inhibitor response can vary by cancer type and disease stage [13]. To our knowledge, no formal criteria for high versus low TMB have yet been established for EMPD specifically. A prior study of 29 EMPD patients reported a median and mean TMB of 5.2 and 6.3 mut/MB, respectively, both higher than that of the patient in the current report [15]. Collectively, these findings suggest that EMPD may respond to immune checkpoint therapy even in the absence of PD-L1 expression by tumor cells, MSI, and high TMB. To what degree expression of antigenpresenting machinery is required remains unclear, as our case demonstrated only minimal surface expression of MHC class I and predominantly cytoplasmic beta-2 microglobulin.

Notably, our report of successful immune checkpoint inhibition for metastatic EMPD represents only a single case. While our findings support further investigation into the safety and efficacy of immune checkpoint inhibitors for this rare and generally fatal metastatic disease, extrapolation of our findings to management of other patients with metastatic EMPD should be conducted with caution. Based on this solitary case, we are unable to provide any estimate of checkpoint inhibitor response rate, and note that even among diseases for which checkpoint inhibitors are FDA-approved, response rates remain modest [7].

\section{Conclusions}

In conclusion, we report the first case to our knowledge of metastatic EMPD with treatment response to immune checkpoint inhibitor therapy, achieving a clinically significant partial response after progression of disease following aggressive chemotherapy. The treatment response was not attributable to known predictors of immune checkpoint

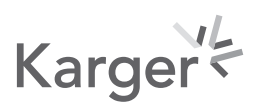


Guercio et al.: Immune Checkpoint Inhibition for Metastatic Extramammary Paget Disease

inhibitor response, such as MSI or high TMB. This case supports further investigation into immunotherapy for metastatic EMPD, a rare clinical entity for which there is no standard treatment.

\section{Acknowledgements}

Not available.

\section{Statement of Ethics}

This study was conducted ethically in accordance with the World Medical Association Declaration of Helsinki. Following the patient's passing, written informed consent was obtained from the patient's next of kin for the publication of this case report and any accompanying images. The institutional review board leadership at Memorial Sloan Kettering Cancer Center concluded that this case report did not require IRB oversight.

\section{Conflict of Interest Statement}

All authors affiliated with Memorial Sloan Kettering Cancer Center report institutional support during the conduct of the study from NIH/NCI Cancer Center Support Grant P30 CA008748, the Pulver family in the form of a charitable donation to Dr. Travis Hollmann, and center support from the Parker Institute for Cancer Immunotherapy at Memorial Sloan Kettering Cancer Center. Dr. Ghafoor and Dr. Chen report no additional disclosures. Dr. Guercio reports support from NIH/NCI Grant T32-CA009207, honoraria from Medscape, and institutional research funding from Bristol-Myers Squibb, Genentech, Eli Lilly, Pfizer, and Sanofi, outside the submitted work. Dr. Hollmann reports research funding from General Electric, outside the submitted work. Dr. Iyer reports grants and personal fees from Mirati Therapeutics, grants from Novartis, grants from DeBioPharm, grants and personal fees from Janssen, outside the submitted work. Dr. Lacouture reports royalties from Legacy Healthcare Services, Apricity Health, LLC, Azitra, Inc., Deciphera, Galderma Research and Development, Johnson and Johnson, NCODA, Novocure Inc., Kyowa Kirin, Inc., Loxo, Merck Sharp and Dohme Corporation, Janssen Research \& Development, LLC, Menlo Therapeutics, Novartis Pharmaceuticals Corporation, QED Therapeutics, F. Hoffmann-La Roche AG, Amgen Inc., AstraZeneca Pharmceuticals LP, Genentech, Inc., Seattle Genetics, Lutris, Paxman Coolers, OnQuality Pharmaceuticals Ltd., Takeda Millenium, outside the submitted work. Dr. Rossi reports personal fees from Regeneron and Biofrontera for advisory board positions, outside the submitted work. Dr. Busam reports royalties from Elsevier. Dr. Janjigian reports stock options from Rgenix, consulting/advisory for AstraZeneca, consulting/advisory for Daiichi Sankyo, research funding from ONO Pharma, consulting/advisory and research funding from Merck \& Co Inc., consulting/advisory and research funding from Bristol-Myers Squibb, research funding from Boehringer Ingelheim, research funding from Bayer, research funding from Genentech/Roche, consulting/advisory for Merck Serono, consulting/advisory for Pfizer, consulting/advisory for Imugene, consulting/advisory and research funding from Eli Lilly, speaking fees from ASCO, consulting/advisory for Michael J. Hennessy Associates, consulting/ advisory for Paradigm Medical Communications LLC, consulting/advisory for Zymeworks Inc., consulting/advisory for Jounce Therapeutics, and consulting/advisory for Seattle Genetics, outside the submitted work. Dr. Jhaveri reports consulting/advisory role and

\section{Karger'}


expenses from AstraZeneca, consulting/advisory, expenses, and institutional research funding from Pfizer, consulting/advisory role and institutional research funding from Novartis, consulting/advisory role and expenses from Taiho Pharmaceutical, advisory board member role for Juno Therapeutics, consulting/advisory role and institutional research funding from ADC Therapeutics, advisory board member role for and institutional research funding from Genentech, consulting/advisory role for Synthon, consulting/advisory role, speaker fees, and expenses from Intellisphere, consultant/advisory role for Spectrum Pharmaceuticals, institutional research funding from Zymeworks, consulting/advisory role and institutional research funding from Eli Lilly and Co, institutional research funding from Novita Pharmaceuticals, institutional research funding from Debio Pharmaceuticals, consulting/ advisory role for Abbvie, consulting/advisory role for Bristol-Myers Squibb, consulting/ advisory role for and expenses from Jounce Therapeutics, consulting/advisory role for Spectrum Pharmaceuticals, institutional research funding from Clovis Oncology, institutional research funding from Immunomedics, and institutional research funding from Puma Biotechnology, outside the submitted work. Dr. Feldman reports research funding and drug supply from Seattle Genetics, research funding from Novartis, research funding and drug supply for clinical trial from Astellas, research funding from Decibel, outside the submitted work. Dr. Bajorin reports honoraria from Merck Sharp \& Dohme, consulting/advisory role, expenses, and institutional research funding from Genentech/Roche, consulting/advisory role, expenses, and institutional research funding from Bristol-Myers Squibb, consulting/advisory role for Fidia Farmaceutici, consulting/advisory role for Pfizer, institutional research funding from AstraZeneca, consulting/advisory role and expenses from Eli Lilly, grants, consulting/ advisory role, expenses, and institutional research funding from Merck, grants, consulting/ advisory role, and institutional research funding from Novartis, consulting/advisory role and expenses from UroGen Pharma, consulting/advisory role for Roche, consulting/advisory role for EMD Serono, institutional research funding from Seattle Genetics, and institutional research funding from Astellas Pharma, outside the submitted work. Dr. Rosenberg reports honoraria, consulting/advisory role, and expenses from Bristol-Myers Squibb, institutional research funding, honoraria, and consulting/advisory role from AstraZeneca, honoraria from Chugai Pharma, consulting/advisory role for Lilly, consulting/advisory role for Merck, consulting/advisory role and institutional research funding from Agensys, consulting/ advisory role, expenses, and institutional research funding from Genentech/Roche, consulting/advisory role for Sanofi, consulting/advisory role for EMD Serono, consulting/ advisory role and institutional research funding from Seattle Genetics, consulting/advisory role and institutional research funding from Bayer, consulting/advisory role for Inovio Pharmaceuticals, consulting/advisory role for BioClin Therapeutics, consulting/advisory role and institutional research funding from QED Therapeutics, consulting/advisory role for Adicet Bio, consulting/advisory role for Sensei Biotherapeutics, consulting/advisory role for Fortress Biotech, consulting/advisory role for Pharmacyclics, consulting/advisory role for Western Oncolytics, consulting/advisory role for GlaxoSmithKline, consulting/advisory role for Janssen Oncology, consulting/advisory role and institutional research funding from Astellas Pharma, consulting/advisory role for Boehringer Ingelheim, consulting/advisory role and institutional research funding from Mirati Therapeutics, institutional research funding from Oncogenex, institutional research funding from Novartis, institutional research funding from Viralytics, institutional research funding from Incyte, institutional research funding from Jounce Therapeutics, stock/ownership interests from Illumina, consulting/advisory role for AstraZeneca/MedImmune, and consulting for Pfizer, outside the submitted work. In addition, Dr. Rosenberg has a patent Predictor of platinum sensitivity owned by Memorial Sloan Kettering Cancer Center. Dr. Hollmann reports research funding from General Electric, outside the submitted work. Dr. Funt reports stock or other ownership interests from Allogene Ther-

\section{Karger'}


apeutics, Urogen, Neogene Therapeutics, Vaxigene, Kronos Bio, and Vida Ventures, research funding and travel expenses from AstraZeneca, research funding from Genentech/Roche, consulting/advisory role for Decibel, and consulting/advisory role for Immunai, outside the submitted work. Dr. Kidwai and Dr. Assis have nothing to disclose.

\section{Funding Sources}

This work was supported by the NIH/NCI Cancer Center Support Grant P30 CA008748, NIH/NCI Grant T32-CA009207, the Pulver family, and the Parker Institute for Cancer Immunotherapy at Memorial Sloan Kettering Cancer Center. T.J.H., a member of the Parker Institute for Cancer Immunotherapy at Memorial Sloan Kettering Cancer Center, participated in the design, analysis, writing, and publication of this manuscript. The remaining sponsors played no role in the design, analysis, writing and publication of the manuscript.

\section{Author Contributions}

All authors contributed to the writing of the manuscript and read and approved the final manuscript. S.A.F., W.Z.K., M.E.L., D.N.A., A.C., V.F., Y.-B.C., K.J.B., Y.Y.J., K.J., D.R.F., and D.F.B. contributed to the clinical care of the patient and provided clinical information. B.J.G., A.M.R., and J.E.R. contributed to the design, analysis, and writing of the manuscript. S.G. created figures depicting radiologic images. G.I. advised on interpretation and presentation of tumor sequencing data. T.J.H. performed investigational immunohistochemical staining of the patient's tumor specimen and created the associated histology figure for publication.

\section{References}

1 Fukuda K, Funakoshi T. Metastatic extramammary Paget's disease: pathogenesis and novel therapeutic approach. Front Oncol. 2018;8:38.

2 van der Linden M, Meeuwis KA, Bulten J, Bosse T, van Poelgeest MI, de Hullu JA. Paget disease of the vulva. Crit Rev Oncol Hematol. 2016;101:60-74.

3 Tanese K, Nakamura Y, Hirai I, Funakoshi T. Updates on the systemic treatment of advanced non-melanoma skin cancer. Front. Med. 2019;6:160.

4 Shah MA, Janjigian YY, Stoller R, Shibata S, Kemeny M, Krishnamurthi S, et al. Randomized multicenter phase II study of modified docetaxel, cisplatin, and fluorouracil (DCF) versus DCF plus growth factor support in patients with metastatic gastric adenocarcinoma: a study of the US Gastric Cancer Consortium. J Clin Oncol. 2015;33(33):3874-9.

5 US Food and Drug Administration. FDA unveils a streamlined path for the authorization of tumor profiling tests alongside its latest product action [updated 2017 Nov 15; cited 2020 Nov 14]. Available from: https:// www.fda.gov/NewsEvents/Newsroom/PressAnnouncements/ucm585347.htm.

6 Andre F, Ciruelos E, Rubovszky G, Campone M, Loibl S, Rugo HS, et al. Alpelisib for PIK3CA-mutated, hormone receptor-positive advanced breast cancer. N Engl J Med. 2019;380(20):1929-40.

7 Tray N, Weber JS, Adams S. Predictive Biomarkers for Checkpoint Immunotherapy: Current Status and Challenges for Clinical Application. Cancer Immunol Res. 2018;6(10):1122-8.

8 Pourmaleki M, Young JH, Socci ND, Chiang S, Edelweiss M, Li Y, et al. Extramammary Paget disease shows differential expression of B7 family members B7-H3, B7-H4, PD-L1, PD-L2 and cancer/testis antigens NY-ESO-1 and MAGE-A. Oncotarget. 2019;10(58):6152-67.

9 Mauzo SH, Tetzlaff MT, Milton DR, Siroy AE, Nagarajan P, Torres-Cabala CA, et al. Expression of PD-1 and PD-L1 in extramammary Paget disease: implications for immune-targeted therapy. Cancers (Basel). 2019;11(6).

10 Karpathiou G, Chauleur C, Hathroubi S, Habougit C, Peoc'h M. Expression of CD3, PD-L1 and CTLA-4 in mammary and extra-mammary Paget disease. Cancer Immunol Immunother. 2018;67(8):1297-303.

11 Urata K, Kajihara I, Miyauchi H, Mijiddorj T, Otsuka-Maeda S, Sakamoto R, et al. The Warburg effect and tumour immune microenvironment in extramammary Paget's disease: overexpression of lactate dehydrogenase A correlates with immune resistance. J Eur Acad Dermatol Venereol. 2019. 
12 Rodig SJ, Gusenleitner D, Jackson DG, Gjini E, Giobbie-Hurder A, Jin C, et al. MHC proteins confer differential sensitivity to CTLA-4 and PD-1 blockade in untreated metastatic melanoma. Sci Transl Med. 2018;10(450).

13 Fernandez EM, Eng K, Beg S, Beltran H, Faltas BM, Mosquera JM, et al. Cancer-specific thresholds adjust for whole exome sequencing-based tumor mutational burden distribution. JCO Precis Oncol. 2019;3.

14 Kang Z, Xu F, Zhu Y, Fu P, Zhang QA, Hu T, et al. Genetic analysis of mismatch repair genes alterations in extramammary Paget disease. Am J Surg Pathol. 2016;40(11):1517-25.

15 Tse J, Elvin JA, Vergilio J-A, Killian JK, Ngo N, Lin DI, et al. Extra-mammary Paget's disease (EMPD) of the skin: A comprehensive genomic profiling (CGP) study. J Clin Oncol. 2019;37(15_Suppl):9591. 